\section{(6) OPEN ACCESS}

${ }^{1}$ Department of Cardiology, Academic Medical CenterUniversity of Amsterdam, Amsterdam, The Netherlands ${ }^{2}$ MRC Epidemiology Unit, Institute of Metabolic Science, Addenbrooke's Hospital, Cambridge, UK

${ }^{3}$ Department of Public Health and Primary Care, Institute of Public Health, University of Cambridge, Cambridge, UK

\section{Correspondence to}

Harald Thune Jørstad, Department of Cardiology, Academic Medical Center, University of Amsterdam, P.O. Box 22660, Amsterdam 1100 DD, The Netherlands; h.t. jorstad@amc.uva.nl

Received 11 February 2015 Revised 12 July 2015 Accepted 14 July 2015 Published Online First 10 August 2015

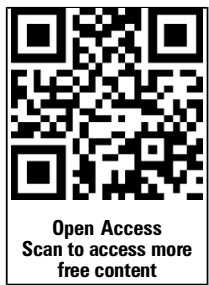

\section{SLinked}

- http://dx.doi.org/10.1136/ heartjnl-2015-308405

\section{CrossMark}

$$
\begin{aligned}
& \text { To cite: Jørstad HT, } \\
& \text { Colkesen EB, Boekholdt SM, } \\
& \text { et al. Heart 2016;102: }
\end{aligned}
$$$$
\text { 63-68. }
$$

\title{
Estimated 10-year cardiovascular mortality seriously underestimates overall cardiovascular risk
}

\begin{abstract}
Objective The European Society of Cardiology's prevention guideline suggests that the risk of total (fatal plus non-fatal) cardiovascular disease (CVD) may be calculated from the risk of CVD mortality using a fixed multiplier (3x). However, the proposed multiplier has not been validated. We investigated the ratio of total CVD to CVD mortality in a large population-based cohort.

Methods CVD mortality and total CVD (fatal plus nonfatal CVD requiring hospitalisation) were analysed using Kaplan-Meier estimates among 24014 men and women aged 39-79 years without baseline CVD or diabetes mellitus in the prospective population-based European Prospective Investigation of Cancer and Nutrition-Norfolk cohort. CVD outcomes included death and

hospitalisations for ischaemic heart disease, heart failure, cerebrovascular disease, peripheral artery disease or aortic aneurysm. The main study outcome was the ratio of 10-year total CVD to 10-year CVD mortality stratified by age and sex.

perspective, total CVD risk is the most relevant parameter for initiating CVD prevention, ${ }^{4}$ and using CVD mortality only can result in underestimation of the total CVD burden. ${ }^{5}$ Although mortality is a more robust clinical outcome, cardiovascular morbidity is equally relevant to providers of healthcare, policy makers and insurance companies. Currently, the relationship between total CVD and CVD mortality in the general population is unclear, and the proposed multiplier for conversion from CVD mortality to total CVD has not been validated.

We hypothesised that the ratio of total CVD (fatal and non-fatal events) and CVD mortality is dependent on age and sex. We tested this hypothesis in the European Prospective Investigation of Cancer and Nutrition-Norfolk (EPIC-Norfolk), a large prospective population-based cohort, with detailed information on various chronic diseases, including CVD mortality and morbidity.
\end{abstract}

Results Ten year CVD mortality was 3.9\% (900 CVD deaths, $95 \% \mathrm{Cl} 3.6 \%$ to $4.1 \%$ ); the rate of total CVD outcomes was $21.2 \%$ (4978 fatal or non-fatal CVD outcomes, $95 \% \mathrm{Cl} 20.7 \%$ to $21.8 \%$ ). The overall ratio of total CVD to CVD mortality was 5.4. However, we found major differences in this ratio when stratified by gender and age. In young women (39-50 years), the ratio of total CVD to CVD mortality was 28.5 , in young men (39-50 years) 11.7. In the oldest age group, these ratios were considerably lower (3.2 in women and 2.4 in men aged 75-79 years).

Conclusions The relationship between 10-year total CVD and CVD mortality is dependent on age and sex, and cannot be estimated using a fixed multiplier. Using CVD mortality to estimate total CVD risk leads to serious underestimation of risk, particularly in younger age groups, and particularly in women.

\section{INTRODUCTION}

The most recent ESC guidelines on cardiovascular disease (CVD) prevention suggest that there is a fixed relationship between CVD mortality and the total burden of CVD events, defined as the composite of fatal and non-fatal CVD. ${ }^{12}$ It is suggested that in high-risk individuals with a 10 -year CVD mortality risk of $\geq 5 \%$, as estimated using Systematic COronary Risk Evaluation (SCORE), total CVD is threefold higher, and possibly more in young men, and less in women and in older individuals. ${ }^{13}$ This has led to the suggestion of using a fixed multiplier $(3 \times)$ for calculating total CVD based on CVD mortality only. From a patient's

\section{METHODS}

\section{Source population}

We used data from the EPIC-Norfolk prospective population study, a cohort of 25639 men and women aged 39-79 years residing in the county of Norfolk, UK. Details of the study have been described elsewhere. ${ }^{6}$ In brief, between 1993 and 1997, 77630 adults were invited from general practices to participate in the study. Of these, 25639 (33\%) provided signed informed consent for study participation and attended a baseline health assessment. Participants completed questionnaires about their personal and family history of disease, drug use and lifestyle, including smoking. Participants were also asked whether a doctor had ever told them that they had any of the following conditions: diabetes mellitus, myocardial infarction or stroke. Anthropometric and blood pressure measurements were performed and non-fasting blood samples were collected at the health assessment. The EPIC-Norfolk cohort was similar to a nationally representative sample for anthropometric indices, blood pressure measurements and serum lipid levels, but with a lower proportion of smokers. ${ }^{6}$ The participants' National Health Service number was used to determine their hospital stay through the East Norfolk Health Authority database, which records all hospital contacts throughout England and Wales for Norfolk residents. Vital status for all EPIC-Norfolk participants was obtained through death certification at the Office for National Statistics. The underlying 
cause of death or hospital admission was coded by trained nosologists according to the International Classification of Diseases (ICD), Tenth Revision. The EPIC-Norfolk study complies with the Declaration of Helsinki. ${ }^{6}$

\section{Study design}

For this analysis, the study population consisted of all EPIC-Norfolk participants who did not report a history of diabetes mellitus, myocardial infarction or stroke at the baseline health assessment. We excluded individuals with diabetes mellitus, as diabetes mellitus is not included as a variable in the SCORE algorithm. CVD mortality was defined as death where CVD was reported as the underlying cause of death on the death certificate. Total CVD was defined as CVD mortality plus hospitalisation with CVD as the underlying cause. Previous validation studies in this cohort indicated high specificity of such case ascertainment. ${ }^{7}$ We defined cardiovascular events or disease as the combination of ischaemic heart disease (ICD codes I20I25), cardiac failure (ICD codes I11, I13, I50), cerebrovascular disease (ICD I60-I69), peripheral artery disease (ICD I70-I79) and aortic aneurysm (ICD I71). We defined 30-day CVD mortality as CVD mortality within 30 days of hospitalisation for a first non-fatal CVD event. CVDs or events not requiring hospitalisation, such as stable angina pectoris, heart failure without hospitalisation or intermittent claudication, were not included in our analysis. We report results for follow-up up to $31 \mathrm{March}$ 2008, a mean follow-up of 11 years.

\section{Statistical methods}

Baseline characteristics were summarised separately for men and women, using numbers and percentages for categorical data, means, 95\% CI and SD for continuous data with a normal distribution, and median and IQR for continuous variables with a non-normal distribution. Ten-year rates of CVD mortality and total CVD were estimated using the Kaplan-Meier (KM) method. Ratios and differences between cardiovascular mortality and morbidity rates were calculated for the total population and in age groups (39-50 years, 50-55 years, 55-60 years, 6065 years, 65-70 years, 70-75 years and 75-79 years), for men and women separately and according to SCORE $(<5 \%, \geq 5 \%)$. We evaluated the calculated total CVD/CVD mortality ratios, including 95\% CIs, by performing individual resampling bootstrapping with 1000 iterations with the same sample size as the original sample. SCORE was calculated using the algorithm for low-risk countries in individuals younger than 65 years, using age at baseline, sex, smoking status, total cholesterol and systolic blood pressure. SCORE was only calculated in individuals with a complete data set of the abovementioned variables. Statistical analyses were performed in SPSS V.21 and STATA V.12.

\section{RESULTS}

A total of 25639 individuals attended the baseline visit. Of these participants, 1625 had diabetes mellitus or a history of vascular disease. The study population consisted of 24014 men and women without prevalent CVD or diabetes mellitus. Table 1 shows baseline characteristics of the study participants. In total, $56.2 \%$ of the study participants were women. Mean age was 58.8 (SD 9.3) years, and $11.8 \%$ were current smokers. Mean values for body mass index, total cholesterol and lowdensity lipoprotein (LDL) cholesterol were slightly above levels recommended in primary prevention setting, respectively, at $26.3 \mathrm{~kg} / \mathrm{m}^{2}$ (SD 3.9) and $6.2 \mathrm{mmol} / \mathrm{L}$ (SD 1.2) and $4.0 \mathrm{mmol} / \mathrm{L}$ (SD 1.1). There were no clinically relevant differences in CVD risk factors between men and women.

Figure 1 shows the 10-year KM curves for cardiovascular mortality and morbidity. A total of 4978 study participants died of or were hospitalised for CVD, yielding a 10 -year cumulative event rate for total CVD of $21.2 \%$ (95\% CI $20.7 \%$ to $21.8 \%$ ). A total of 900 study participants died of a CVD or event, yielding a 10 -year CVD mortality rate of $3.9 \%$ (95\% CI $3.6 \%$ to 4.1\%). The overall ratio of total CVD/CVD mortality was 5.4. Of the 4978 study participants with a CVD or event, 360 individuals had a fatal event as first event (7.2\% of total CVD); when 30-day CVD mortality was included this number was 643 (12.9\% of total CVD). Of the 4618 non-fatal CVD events/hospitalisations, the majority was ischaemic heart disease $(45.6 \%)$ followed by peripheral arterial disease $(19.7 \%)$ and congestive heart failure $(16.9 \%)$. Only $2.9 \%$ of the non-fatal events/hospitalisations were caused by an aortic aneurysm (table 2).

Table 3 presents 10 -year CVD mortality and total CVD by age and sex. In men, the KM estimate for total CVD was $24.9 \%$, for CVD mortality this was $5.4 \%$, yielding an overall ratio of 4.6. In women, total CVD was $18.4 \%$, CVD mortality 2.7\%, with an overall ratio of 6.8. Among 2219 men aged 3950 years, the KM estimate for total CVD was $8.2 \%$ and for CVD mortality $0.7 \%$, resulting in a ratio of 11.7 . Among 3061

Table 1 Population characteristics

\begin{tabular}{|c|c|c|c|}
\hline Population characteristics $(n=24014)$ & $\begin{array}{l}\text { Total } \\
(n=24014)\end{array}$ & $\begin{array}{l}\text { Male } \\
(n=10509)\end{array}$ & $\begin{array}{l}\text { Female } \\
(n=13505)\end{array}$ \\
\hline Age, years & $58.8 \pm 9.3$ & $59.0 \pm 9.3$ & $58.7 \pm 9.3$ \\
\hline Weight, kg & $73.3 \pm 13.1$ & $80.3 \pm 11.4$ & $67.9 \pm 11.8$ \\
\hline Body mass index, $\mathrm{kg} / \mathrm{m}^{2}$ & $26.3 \pm 3.9$ & $26.4 \pm 3.3$ & $26.2 \pm 4.3$ \\
\hline Waist/hip ratio & $0.85 \pm 0.09$ & $0.93 \pm 0.06$ & $0.79 \pm 0.06$ \\
\hline Current smokers & $2836(11.8)$ & $1297(12.3)$ & 1539 (11.4) \\
\hline Systolic blood pressure, mm Hg & $135.2 \pm 18.3$ & $137.1 \pm 17.5$ & $133.7 \pm 18.8$ \\
\hline Diastolic blood pressure, $\mathrm{mm} \mathrm{Hg}$ & $82.4 \pm 11.2$ & $84.4 \pm 11.1$ & $80.9 \pm 11.1$ \\
\hline Total cholesterol, $\mathrm{mmol} / \mathrm{L}$ & $6.2 \pm 1.2$ & $6.0 \pm 1.1$ & $6.3 \pm 1.1$ \\
\hline LDL cholesterol, mmol/L & $4.0 \pm 1.0$ & $3.9 \pm 1.0$ & $4.0 \pm 1.1$ \\
\hline HDL cholesterol, mmol/L & $1.4 \pm 0.4$ & $1.2 \pm 0.3$ & $1.6 \pm 0.4$ \\
\hline Triglycerides, mmol/L & $1.5(1.1-2.2)$ & $1.7(1.2-2.5)$ & $1.4(1.0-2.0)$ \\
\hline SCORE, \% ( $n=15$ 171) & $1.55 \pm 1.8$ & $2.35 \pm 2.2$ & $0.95 \pm 1.1$ \\
\hline
\end{tabular}

Data are presented as number (percentage), mean \pm SD or median (IQR).

HDL, high-density lipoprotein; LDL, low-density lipoprotein; SCORE, Systematic Coronary Risk Evaluation, expressed as estimated 10-year mortality risk. 


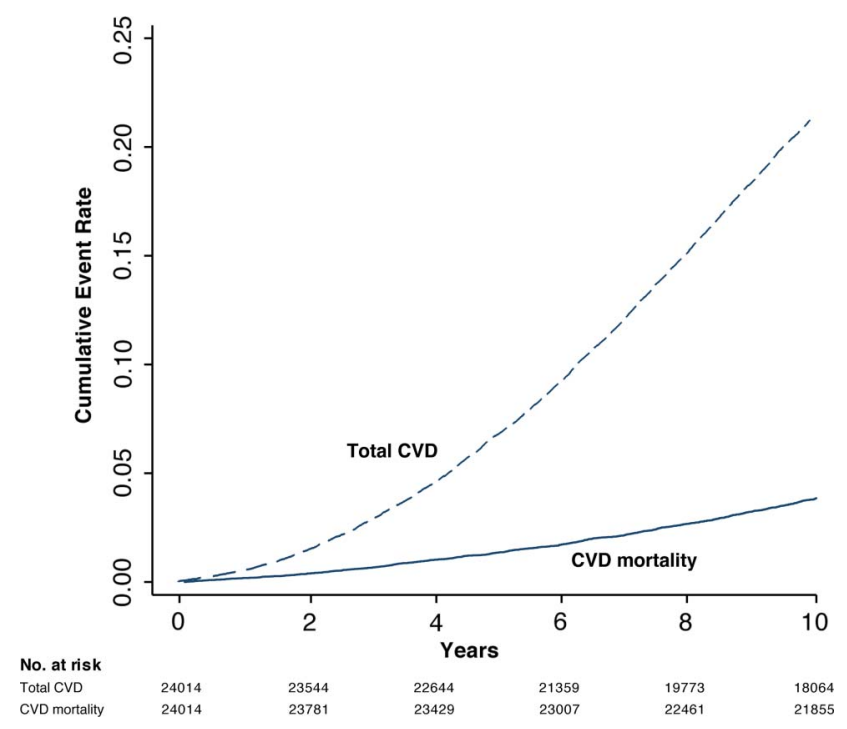

Figure 1 Kaplan-Meier estimates of the 10-year cumulative total cardiovascular disease (CVD) and CVD mortality. CVD mortality is death from a CVD. Total CVD is all fatal and non-fatal CVD or events requiring hospitalisation.

women aged 39-50 years, total CVD was 5.7\% and CVD mortality $0.2 \%$, resulting in a ratio of 28.5 . Event rates increased with age. Among 328 men aged 75-79 years, total CVD was $56.3 \%$ and CVD mortality $24.9 \%$, amounting to a ratio of 2.4. Among 366 women aged 75-79 years, total CVD was $47.3 \%$ and CVD mortality $14.6 \%$, resulting in a ratio of 3.2. Neither the ratio of nor the difference between total CVD and CVD mortality was constant across categories of age in either sex (table 3, right panels; figure 2). Overall, the total CVD/CVD mortality ratios were inversely related to age, and with greater variation among women as compared with men (figure 2).

SCORE was calculated in 15171 individuals up to 65 years of age as shown in table 4. Mean predicted CVD mortality according to SCORE was $1.55 \%$ (95\% CI $1.52 \%$ to $1.58 \%)$; in men $2.35 \%$ (95\% CI $2.29 \%$ to $2.40 \%$ ) and in women $0.95 \%$ (95\% CI $0.92 \%$ to $0.97 \%$ ). In individuals with $\geq 5 \%$ SCORE, 10-year CVD mortality was 7.3\%, whereas 10-year total CVD was $41.2 \%$, yielding a ratio of 5.6. In men with a SCORE $\geq 5 \%$,

Table 2 Non-fatal 10-year CVD according to type

\begin{tabular}{|c|c|c|c|c|c|c|}
\hline \multirow[b]{2}{*}{ Type of event } & \multicolumn{2}{|l|}{ Total } & \multicolumn{2}{|l|}{ Male } & \multicolumn{2}{|c|}{ Female } \\
\hline & $\mathrm{n}$ & (Per cent) & $\mathbf{n}$ & (Per cent) & $\mathbf{n}$ & (Per cent) \\
\hline $\begin{array}{l}\text { Ischaemic heart } \\
\text { disease }\end{array}$ & 2105 & (45.6) & 1260 & $(46.9)$ & 845 & $(43.8)$ \\
\hline $\begin{array}{l}\text { Congestive heart } \\
\text { failure }\end{array}$ & 781 & (16.9) & 444 & (16.5) & 337 & (17.5) \\
\hline $\begin{array}{l}\text { Cerebrovascular } \\
\text { disease }\end{array}$ & 686 & $(14.9)$ & 332 & (12.4) & 354 & (18.4) \\
\hline Haemorrhagic & 118 & $(2.6)$ & 55 & $(2.1)$ & 63 & (3.3) \\
\hline Ischaemic & 568 & (12.3) & 277 & $(10.3)$ & 291 & $(15.1)$ \\
\hline $\begin{array}{l}\text { Peripheral arterial } \\
\text { disease }\end{array}$ & 912 & (19.7) & 547 & (20.4) & 365 & (18.9) \\
\hline Aortic aneurysm & 134 & (2.9) & 104 & (3.9) & 30 & (1.6) \\
\hline
\end{tabular}

Non-fatal 10-year CVD includes CVD diseases or events requiring hospitalisation. Fatal CVD is not included in the table. Data are presented as number (percentage).

Percentages may not add up to 100 because of rounding.

CVD, cardiovascular disease. this ratio was 5.4 , in women 9.4. In individuals with a SCORE $<5 \%$, these ratios varied considerably: 12.5 in the total population, in men 10.4 , in women 15.9.

Total CVD/CVD mortality ratios as assessed using bootstrap resampling were comparable across the total population and the subset wherein SCORE was calculated. Only in the youngest age group, in women more than men, these ratios were higher when calculated using bootstrap resampling (women aged 39-50 years KM-ratio 28.5 vs bootstrap ratio 36.06 (95\% CI 35.28 to $36.88)$, men aged $39-50$ years KM-ratio 11.7 vs bootstrap ratio 12.21 (95\% CI 12.07 to 12.36$)$ ), most likely related to the low number of mortality events in these subgroups.

\section{DISCUSSION}

Our analysis demonstrates a complex relationship between 10-year total CVD and CVD mortality in the EPIC-Norfolk prospective population study, a large European cohort. Men and women showed a decreasing CVD morbidity/mortality ratio with increasing age, and with a greater ratio for women in all age groups. Thus, our results suggest that the ratios of total CVD/CVD mortality are age-dependent and sex-dependent. Furthermore, only $12.9 \%$ of first CVD events were fatal. By focusing on CVD mortality only, the overall burden of CVD is seriously underestimated, leaving large numbers of individuals untreated, despite the fact that their risk of CVD events is substantial.

The ESC prevention guidelines use the 10-year cardiovascular mortality risk predictor SCORE as a decision-making tool in primary prevention. ${ }^{2}{ }^{8}$ Using SCORE risk charts, clinicians can identify individuals with a high risk $(\geq 5 \%)$ of 10 -year CVD mortality. Based on data from the FINRISK study, it is suggested that at the level at which risk management is recommended $(5 \%$ risk of 10-year cardiovascular mortality), total event risk, that is, including morbidity, is about three times higher (15\%). The guideline suggests this ratio may be used as a multiplier in calculating total CVD risk based on estimated CVD mortality. ${ }^{1-3}$ In our study, the total CVD/CVD mortality ratio in individuals with a SCORE $\geq 5 \%$ was markedly higher (5.6) than the suggested multiplier (3), more so in women (9.4) as compared with men (5.4). Noteworthy is that only 77 women up to 65 years of age had a $\mathrm{SCORE} \geq 5 \%$. Also when not stratified by SCORE, these ratios were overall higher (total population 5.4, in men 4.6 , in women 6.8 ). The ratio was especially high (28.5 in women, 11.7 in men) in the lower age subgroups (39-50 years), and decreased with age. While risk scores such as SCORE identify individuals at high risk of CVD mortality, our findings show that risk of CVD mortality cannot be readily extrapolated to risk of total CVD using a fixed multiplier. A high risk of CVD mortality suggests a high risk of total CVD, regardless of age and sex, with all inherent implications for primary prevention. However, our study shows that a low risk of CVD mortality does not translate into a proportionally low risk of CVD morbidity, particularly in young individuals, and in women more than men. This discrepancy should be taken into account in the clinical decision-making process regarding preventive measures in young patients.

The majority of first non-fatal events or hospitalisations were caused by ischaemic CVD (in total $77.6 \%$ including ischaemic heart disease, ischaemic cerebrovascular disease and peripheral arterial disease). As healthy lifestyles and preventive medication have been shown to significantly reduce the risk for such events, this underlines the need for preventive measures in these individuals. Although CVD mortality is clearly the most robust clinical outcome, cardiovascular morbidity is likely to be at least as 
Table 3 Cumulative 10-year CVD mortality and total CVD by sex and age

\begin{tabular}{|c|c|c|c|c|c|c|c|c|c|c|c|c|}
\hline \multirow[b]{2}{*}{ Sex } & \multicolumn{2}{|c|}{ Age group } & \multicolumn{3}{|c|}{ 10-year CVD mortality } & \multicolumn{3}{|c|}{ 10-year total CVD } & \multirow[b]{2}{*}{ KM difference } & \multirow[b]{2}{*}{$\mathrm{KM}$ ratio } & \multirow[b]{2}{*}{ BSP ratio } & \multirow[b]{2}{*}{$95 \% \mathrm{Cl}$ of $\mathrm{BSP}$ ratic } \\
\hline & & $\mathrm{N}$ & $\mathrm{n}$ & KM rate & $95 \% \mathrm{Cl}$ & $\mathrm{n}$ & KM rate & $95 \% \mathrm{Cl}$ & & & & \\
\hline \multicolumn{13}{|c|}{ Male } \\
\hline & $39-50$ & 2219 & 15 & 0.7 & (0.4 to 1.1 ) & 181 & 8.2 & (7.2 to 9.5$)$ & 7.5 & 11.7 & 12.21 & (12.07 to 12.36$)$ \\
\hline & $50-55$ & 1780 & 26 & 1.5 & (1.0 to 2.2 ) & 260 & 14.8 & (13.2 to 16.5 ) & 13.3 & 9.9 & 10.01 & (9.93 to 10.09 ) \\
\hline & $55-60$ & 1637 & 34 & 2.1 & (1.5 to 3.0 ) & 320 & 19.9 & (18.6 to 22.0 ) & 17.8 & 9.5 & 9.37 & (9.30 to 9.44 ) \\
\hline & $60-65$ & 1633 & 67 & 4.2 & (3.4 to 5.4 ) & 462 & 29.0 & (26.8 to 31.3 ) & 24.8 & 6.9 & 6.84 & (6.81 to 6.87 ) \\
\hline & $65-70$ & 1622 & 127 & 8.3 & (7.0 to 9.8 ) & 565 & 36.5 & ( 34.2 to 39.0 ) & 28.2 & 4.4 & 4.42 & (4.41 to 4.43 ) \\
\hline & $70-75$ & 1290 & 209 & 17.7 & (15.6 to 20.0 ) & 586 & 48.8 & ( 46.0 to 51.7 ) & 31.1 & 2.8 & 2.77 & (2.76 to 2.77 ) \\
\hline & $75-79$ & 328 & 65 & 23.3 & (18.7 to 28.8 ) & 167 & 56.3 & (50.6 to 62.1 ) & 33 & 2.4 & 2.41 & (2.40 to 2.42 ) \\
\hline & Total & 10509 & 543 & 5.4 & (4.9 to 5.8 ) & 2541 & 24.9 & (24.1 to 25.7 ) & 19.5 & 4.6 & 4.66 & (4.65 to 4.67 ) \\
\hline \multicolumn{13}{|c|}{ Female } \\
\hline & $39-50$ & 3061 & 5 & 0.2 & (0.07 to 0.4 ) & 173 & 5.7 & (4.9 to 6.6 ) & 5.5 & 28.5 & 36.06 & (35.28 to 36.88 ) \\
\hline & $50-55$ & 2333 & 11 & 0.5 & (0.3 to 0.9$)$ & 225 & 9.8 & (8.6 to 11.1 ) & 9.3 & 19.6 & 20.24 & (19.95 to 20.55$)$ \\
\hline & $55-60$ & 2129 & 17 & 0.8 & (0.5 to 1.3 ) & 299 & 14.2 & (12.8 to 15.8 ) & 13.4 & 17.8 & 17.65 & (17.44 to 17.87 ) \\
\hline & $60-65$ & 2014 & 43 & 2.2 & (1.6 to 2.9 ) & 395 & 20.1 & (18.4 to 21.9 ) & 17.9 & 9.1 & 9.16 & (9.10 to 9.22$)$ \\
\hline & $65-70$ & 1995 & 86 & 4.5 & (3.6 to 5.5 ) & 556 & 28.8 & (26.8 to 30.9 ) & 24.3 & 6.4 & 6.47 & (6.44 to 6.50$)$ \\
\hline & $70-75$ & 1607 & 145 & 9.5 & ( 8.2 to 11.1 ) & 624 & 40.7 & ( 38.3 to 43.2 ) & 31.2 & 4.3 & 4.28 & ( 4.27 to 4.30 ) \\
\hline & $75-79$ & 366 & 50 & 14.6 & (11.3 to 18.8 ) & 165 & 47.3 & ( 42.2 to 52.7 ) & 32.7 & 3.2 & 3.26 & (3.24 to 3.27 ) \\
\hline & Total & 13505 & 357 & 2.7 & (2.4 to 3.0$)$ & 2437 & 18.4 & (17.8 to 19.1 ) & 15.7 & 6.8 & 6.80 & (6.79 to 6.82 ) \\
\hline
\end{tabular}

CVD mortality is death from a CVD. Total CVD is all fatal and non-fatal CVD or events requiring hospitalisation.

Cumulative total CVD and CVD mortality rates were calculated using the KM method.

KM difference is difference of total CVD and CVD mortality of the KM estimates. KM ratio is the ratio of total CVD to CVD mortality of the KM estimates. BSP ratio is the ratio of total

CVD to CVD mortality of the bootstrap procedure estimates.

BSP, bootstrap procedure; CVD, cardiovascular disease; ${ }_{\text {, }}$ KM, Kaplan-Meier.

relevant to patients, providers of healthcare, policy makers and insurance companies. Currently, $10 \%$ of the global disease burden is attributed to CVD, with CVD being responsible for 151.377 million disability-adjusted life years. ${ }^{9}$ CVD mortality alone contributed to 17.3 million deaths in 2008, representing $30 \%$ of all global deaths, with a projected number of deaths of almost 23.6 million in $2030 .^{9}$ The burden of total CVD is likely to show a similar or even greater increase, as CVD mortality has declined relative to CVD morbidity in recent decades. ${ }^{10}{ }^{11}$ This relative decline in mortality can be attributed to improved acute and chronic CVD treatments, as well as improvements in primary and secondary prevention. Considering the individual, economic and societal implications of CVD morbidity, guidelines could be significantly improved by including morbidity in the estimation of risk.

Different fatal/non-fatal ratios have been published in previous studies, for which several explanations may be found. Similar to our results, van Dis et $a l^{12}$ showed that the ratio of total CVD to CVD mortality was 4.0 in men and 5.2 in women in a large Dutch population. However, they did not present ratios across different age groups. In a number of primary prevention trials in selected populations, the ratios of total CVD to CVD mortality varied between 3.2 and 4.5 for the overall population. ${ }^{13-16}$ In comparison to our study, these trials were conducted in a predominantly male study population, and did not report numbers of events in sex and age subgroups.

In the landmark trials of aspirin use in primary prevention of coronary heart disease, this ratio was 3.7 (fatal CVD vs nonfatal myocardial infarction and stroke) in the US Physicians Health Study, while the British counterpart study showed a ratio of 1.1 (fatal CVD vs non-fatal myocardial infarction). ${ }^{17}$ Both studies used different diagnostic categories which were narrower than those used in our study. It has previously been hypothesised that these differences in ratios reflect diagnostic differences (such as ascertainment and diagnostic thresholds) rather than underlying disease differences. ${ }^{19}$ Furthermore, study power may play a role. Greenland et $a l^{20}$ showed lower ratios of non-fatal myocardial infarction to fatal CVD, especially in the very young (ages 18-39 years men 1.5, women 2.2, ages 40-59 years men 0.8 , women 0.9 ). However, the number of non-fatal outcome events in our study was roughly 10 -fold the number in their study. In our study, we chose to address CVD (all arterial
Figure 2 Ratios of 10-year cumulative total CVD to CVD mortality by sex and age groups. CVD, cardiovascular disease.

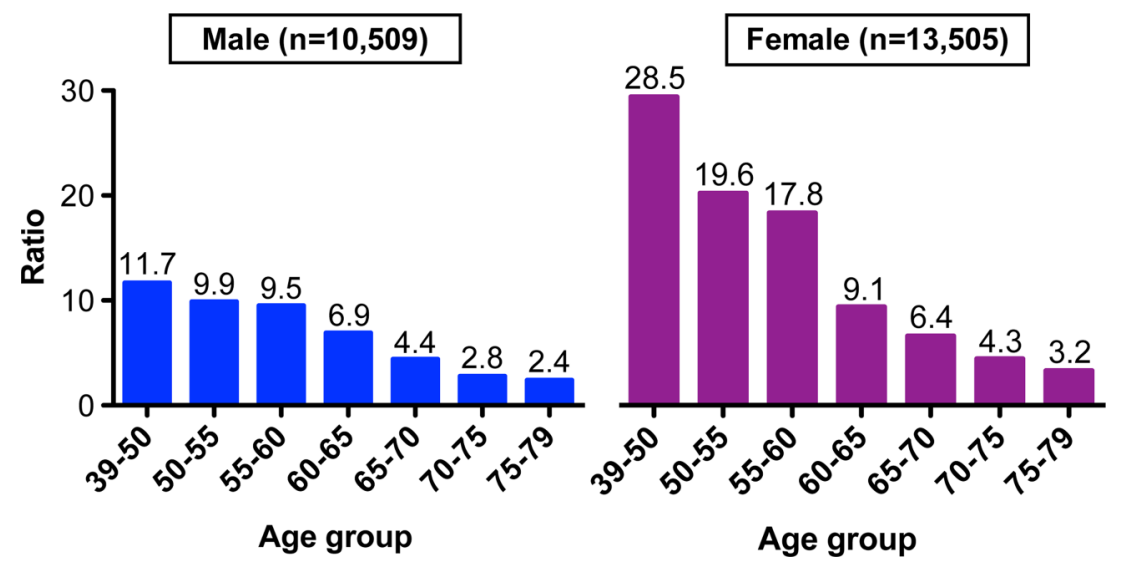


Table 4 Cumulative 10-year CVD mortality and total CVD by sex and SCORE

\begin{tabular}{|c|c|c|c|c|c|c|c|c|c|c|}
\hline \multirow[b]{2}{*}{ Score } & \multirow[b]{2}{*}{$\mathbf{N}$} & \multicolumn{3}{|c|}{ 10-year CVD mortality } & \multicolumn{3}{|c|}{ 10-year total CVD } & \multirow[b]{2}{*}{ KM ratio } & \multirow[b]{2}{*}{ BSP ratio } & \multirow[b]{2}{*}{$95 \% \mathrm{Cl}$} \\
\hline & & n & $\mathrm{KM}$ rate & $95 \% \mathrm{Cl}$ & $n$ & KM & $95 \% \mathrm{Cl}$ & & & \\
\hline \multicolumn{11}{|c|}{ Total population } \\
\hline$<5 \%$ & 14491 & 139 & 1.0 & (0.8 to 1.2$)$ & 1783 & 12.5 & (11.9 to 13.0$)$ & 12.5 & 12.83 & (12.77 to 12.93 ) \\
\hline$\geq 5 \%$ & 680 & 48 & 7.3 & (5.5 to 9.6 ) & 274 & 41.2 & (37.6 to 45.1 ) & 5.6 & 5.68 & (5.65 to 5.72 ) \\
\hline \multicolumn{11}{|l|}{ Male } \\
\hline$<5 \%$ & 5906 & 81 & 1.4 & (1.1 to 1.7 ) & 842 & 14.5 & (13.6 to 15.4$)$ & 10.4 & 10.34 & (10.29 to 10.39 ) \\
\hline$\geq 5 \%$ & 603 & 45 & 7.7 & (5.8 to 10.2 ) & 246 & 41.7 & (37.8 to 45.7 ) & 5.4 & 5.42 & (5.39 to 5.46$)$ \\
\hline \multicolumn{11}{|l|}{ Female } \\
\hline$<5 \%$ & 8585 & 58 & 0.7 & (0.5 to 0.9$)$ & 941 & 11.1 & (10.5 to 11.8$)$ & 15.9 & 16.28 & (16.19 to 16.38 ) \\
\hline$\geq 5 \%$ & 77 & 3 & 4.0 & (1.3 to 11.2 ) & 28 & 37.7 & (27.8 to 49.7 ) & 9.4 & 9.52 & (9.28 to 9.75 ) \\
\hline
\end{tabular}

territories) instead of coronary disease alone, as the SCORE risk factors impact on all arterial territories. In addition, we used a broader definition of non-fatal CVD compared with the study by Greenland $e t a l$, as we believe that the 'milder' forms of nonfatal CVD also represent clinically important CVD. These nonfatal presentations of CVD have implications for symptomatic treatment and preventive measures, and play an important role in healthcare finances and healthcare utilisation.

Currently, most major guidelines focus on 10-year risk estimation, based on different risk algorithms with outcomes including various combinations of fatal and non-fatal events in different arterial territories. $^{21}$ Our results emphasise the limitations of calculating the risk of fatal CVD events in a limited number of arterial territories for a period of 10 years. Lifetime risk instead of 10-year risk may also be used to characterise the relationship between fatal and total CVD. However, with a lifetime of CVD mortality estimated at $26 \%$ in women and $36 \%$ in men (Caucasian, all ages combined), the impact of morbidity may again be underestimated. ${ }^{22}$

Particularly, the addition of substantial numbers of diseasefree years of life is an important goal of preventive therapy. Studies in modern population-based cohorts with lifetime event rates available may better characterise the current and future public burden of CVD, improve decision making on preventive therapy and improve communication of risk between patients and clinicians.

\section{Strengths and limitations}

There are several strengths to our study. First, our analysis was performed in a large, population-based cohort with long-term follow-up and detailed information on mortality and hospitalisations, and we were able to estimate cumulative mortality and hospitalisation rates in the overall population and in large age and sex subgroups. Second, the EPIC-Norfolk cohort is similar to a nationally representative sample for anthropometric variables, blood pressure and serum lipids. ${ }^{6}{ }^{23}$ However, it should be noted that the population in the Norfolk area is healthier than the general UK population with a standardised mortality ratio of 0.94 (source: Office for National Statistics). Third, we have previously shown that the SCORE low-risk algorithm performs better than the high-risk algorithm in prediction 10-year CVD mortality. ${ }^{24}$ The recent reclassification of the UK as a low-risk country in the ESC prevention guidelines is in line with our findings. 24
Some aspects of our study warrant consideration. First, we used data from a prospective cohort, originally designed for the investigation of parameters other than cardiovascular events and hospitalisations, with the limitations inherent in this type of analysis. Second, while we were able to calculate SCORE in a large number of individuals (15 171), there were only 77 women with a SCORE of $\geq 5 \%$, meaning that nearly all women had a SCORE under the threshold where risk management is recommended. These women had a very low rate of fatal CVD, but a considerable risk of non-fatal CVD, contributing to the very high ratios of total/fatal-CVD. This underlines the fact that CVD mortality cannot readily be used to calculate total CVD, and we do not recommended using these ratios to calculate total CVD from fatal CVD. However, these ratios illustrate the limitations of mortality as a single outcome parameter, particularly at young ages and in women, and emphasise the need to include non-fatal outcomes in estimations of the risk of cardiovascular events.

Finally, the definition of CVD events is essential in any study investigating the relationship between total CVD and CVD mortality. We defined total CVD as any event or disease requiring hospitalisation, including ischaemic heart disease, cardiac failure, cerebrovascular disease, peripheral artery disease and aortic aneurysm. CVD not requiring hospitalisation, including 'mild' peripheral artery disease, 'mild' heart failure or stable angina pectoris, was not included in our analysis. While these variants of CVD frequently do not require hospitalisation, they are expressions of clinically relevant atherosclerotic disease and form a relevant indicator for the initiation or intensification of preventive measures in individual patients. Using a broader definition of CVD would result in a higher rate of non-fatal CVD, leading to a further increase in the overall ratios of total CVD/ CVD mortality, and potentially change the threshold for preventive therapy.

\section{Conclusion}

In summary, among patients without a history of CVD or diabetes mellitus, the relation between total CVD including hospitalisations and CVD mortality is highly variable. The total CVD to CVD mortality ratio is inversely related to age, and is higher in women as compared with men. Our findings do not support using a fixed multiplier to calculate total CVD risk based on CVD mortality risk, and caution is warranted when extrapolating the risk of CVD mortality to the risk of total CVD. Future guidelines may be revised to reflect these relationships. 


\section{Key messages}

What is already known on this subject?

- The European Society of Cardiology's Prevention Guideline suggests that total (fatal and non-fatal) cardiovascular disease (CVD) risk may be calculated from the risk of CVD mortality by using a fixed multiplier $(3 x)$

- The relationship between total CVD and CVD mortality in the general population is unclear, and the suggested fixed multiplier has not been validated

\section{What might this study add?}

- The relationship between 10-year total CVD and CVD mortality is dependent on age and sex, and cannot be estimated using a fixed multiplier

- Using CVD mortality to estimate total CVD risk leads to serious underestimation of risk, particularly in younger age groups, and particularly in women

\section{How might this impact on clinical practice?}

- These findings have major implications for individual decision making on preventive therapy and for future guidelines on prevention

- A significantly greater proportion of individuals will benefit from preventive treatments than is estimated by current European Society of Cardiology guidelines, particularly in young age groups

Acknowledgements The authors thank the participants, general practitioners and staff in EPIC-Norfolk.

Contributors All authors contributed to the planning, conduct and reporting of the study.

Funding EPIC-Norfolk is supported by programme grants from the Medical Research Council UK (MRC G0401527, MRC G0701863, MRC G1000143) and Cancer Research UK (CRUK 8257).

Competing interests None declared.

Ethics approval Norfolk Local Research Ethics Committee.

Provenance and peer review Not commissioned; externally peer reviewed.

Data sharing statement Requests for additional data can be directed to the corresponding author.

Open Access This is an Open Access article distributed in accordance with the Creative Commons Attribution Non Commercial (CC BY-NC 4.0) license, which permits others to distribute, remix, adapt, build upon this work non-commercially, and license their derivative works on different terms, provided the original work is properly cited and the use is non-commercial. See: http://creativecommons.org/ licenses/by-nc/4.0/

\section{REFERENCES}

1 Graham I, Atar D, Borch-Johnsen K, et al. European guidelines on cardiovascular disease prevention in clinical practice: executive summary: Fourth Joint Task Force of the European Society of Cardiology and Other Societies on Cardiovascular Disease
Prevention in Clinical Practice (Constituted by representatives of nine societies and by invited experts). Eur Heart I 2007;28:2375-414.

2 Perk J, De Backer G, Gohlke H, et al. European Guidelines on cardiovascular disease prevention in clinical practice (version 2012). Eur Heart J 2012;33: 1635-701.

3 Vartiainen $E$, Jousilahti $P$, Alfthan $G$, et al. Cardiovascular risk factor changes in Finland, 1972-1997. Int J Epidemiol 2000;29:49-56.

4 Cooney MT, Dudina A, D'Agostino R, et al. Cardiovascular risk-estimation systems in primary prevention: do they differ? Do they make a difference? Can we see the future? Circulation 2010;122:300-10.

5 D'Agostino RB, Vasan RS, Pencina MJ, et al. General cardiovascular risk profile for use in primary care: the Framingham Heart Study. Circulation 2008;117:743-53.

6 Day N, Oakes S, Luben R, et al. EPIC-Norfolk: study design and characteristics of the cohort. European Prospective Investigation of Cancer. Br I Cancer 1999;80 (Suppl 1):95-103.

7 Boekholdt SM, Peters RJG, Day NE, et al. Macrophage migration inhibitory factor and the risk of myocardial infarction or death due to coronary artery disease in adults without prior myocardial infarction or stroke: the EPIC-Norfolk Prospective Population study. Am J Med 2004;117:390-7.

8 Conroy RM, Pyörälä K, Fitzgerald AP, et al. Estimation of ten-year risk of fatal cardiovascular disease in Europe: the SCORE project. Eur Heart J 2003:24:987-1003.

9 Mendis S, Puska P, Norrving B. Global atlas on cardiovascular disease prevention and control. World Health Organization, 2011.

10 Tunstall-Pedoe $\mathrm{H}$, Kuulasmaa $\mathrm{K}$, Mähönen $\mathrm{M}$, et al. Contribution of trends in survival and coronary-event rates to changes in coronary heart disease mortality: 10-year results from 37 WHO MONICA project populations. Monitoring trends and determinants in cardiovascular disease. Lancet 1999;353:1547-57.

11 Hardoon SL, Whincup PH, Petersen I, et al. Trends in longer-term survival following an acute myocardial infarction and prescribing of evidenced-based medications in primary care in the UK from 1991: a longitudinal population-based study. J Epidemiol Community Health 2011;65:770-4.

12 van Dis I, Geleijnse JM, Boer JM, et al. Effect of including nonfatal events in cardiovascular risk estimation, illustrated with data from The Netherlands. Eur I Prev Cardiol 2014:377-83

13 Colhoun HM, Betteridge DJ, Durrington PN, et al. Primary prevention of cardiovascular disease with atorvastatin in type 2 diabetes in the Collaborative Atorvastatin Diabetes Study (CARDS): multicentre randomised placebo-controlled trial. Lancet 2004;364:685-96

14 Collaboration CTTC, Baigent C, Blackwell L, et al. Efficacy and safety of more intensive lowering of LDL cholesterol: a meta-analysis of data from 170,000 participants in 26 randomised trials. Lancet 2010;376:1670-81.

15 Ridker PM, Danielson E, Fonseca FAH, et al. Rosuvastatin to prevent vascular events in men and women with elevated C-reactive protein. N Engl J Med 2008;359: 2195-207.

16 Shepherd J, Cobbe SM, Ford I, et al. Prevention of coronary heart disease with pravastatin in men with hypercholesterolemia. West of Scotland Coronary Prevention Study Group. N Engl I Med 1995;333:1301-7.

17 Final report on the aspirin component of the ongoing Physicians' Health Study. Steering Committee of the Physicians' Health Study Research Group. N Engl JMed 1989:321:129-35.

18 Peto R, Gray R, Collins R, et al. Randomised trial of prophylactic daily aspirin in British male doctors. Br Med I (Clin Res Ed) 1988:296:313-16.

19 Khaw K-T. Correspondence: physicians' health study: aspirin and primary preventions of coronary heart disease. N Engl J Med 1989:321:1825-6.

20 Greenland P, Knoll MD, Stamler J, et al. Major risk factors as antecedents of fatal and nonfatal coronary heart disease events. JAMA 2003;290:891-7.

21 Ferket BS, Colkesen EB, Visser JJ, et al. Systematic review of guidelines on cardiovascular risk assessment: Which recommendations should clinicians follow for a cardiovascular health check? Arch Intern Med 2010;170:27-40.

22 Berry JD, Dyer A, Cai X, et al. Lifetime risks of cardiovascular disease. N Engl J Med 2012:366:321-9.

23 Bennett N, Dodd T, Flatley F, et al. Health survey for England 1993. London: HMSO, 1995.

24 Jørstad HT, Colkesen EB, Minneboo M, et al. The Systematic COronary Risk Evaluation (SCORE) in a large UK population: 10-year follow-up in the EPIC-Norfolk prospective population study. Eur J Prev Cardiol 2015;22:119-26. 